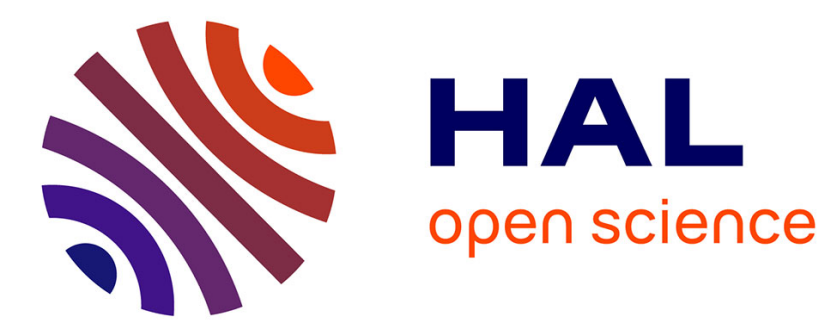

\title{
Non-circular algebraic curves of constant width: an answer to Rabinowitz
}

\author{
Yves Martinez-Maure
}

\section{To cite this version:}

Yves Martinez-Maure. Non-circular algebraic curves of constant width: an answer to Rabinowitz. 2021. hal-03265440

\author{
HAL Id: hal-03265440 \\ https://hal.science/hal-03265440
}

Preprint submitted on 20 Jun 2021

HAL is a multi-disciplinary open access archive for the deposit and dissemination of scientific research documents, whether they are published or not. The documents may come from teaching and research institutions in France or abroad, or from public or private research centers.
L'archive ouverte pluridisciplinaire HAL, est destinée au dépôt et à la diffusion de documents scientifiques de niveau recherche, publiés ou non, émanant des établissements d'enseignement et de recherche français ou étrangers, des laboratoires publics ou privés. 


\title{
Non-circular algebraic curves of constant width: an answer to Rabinowitz
}

Yves Martinez-Maure

yves.martinez-maure@imj-prg.fr

\begin{abstract}
In response to an open problem raised by S. Rabinowitz, we prove that

$$
\begin{aligned}
& \left(\left(x^{2}+y^{2}\right)^{2}+8 y\left(y^{2}-3 x^{2}\right)\right)^{2}+432 y\left(y^{2}-3 x^{2}\right)\left(351-10\left(x^{2}+y^{2}\right)\right) \\
& =567^{3}+28\left(x^{2}+y^{2}\right)^{3}+486\left(x^{2}+y^{2}\right)\left(67\left(x^{2}+y^{2}\right)-567 \times 18\right)
\end{aligned}
$$
\end{abstract}

is the equation of a plane convex curve of constant width.

\section{Introduction}

A disk has the property that it can be rotated between two fixed parallel lines without losing contact with either line. It has been known for a long time that there are many other plane convex bodies with the same property. Such plane convex bodies are called plane convex bodies 'of constant width' or 'orbiforms'. Their boundaries are of course called 'plane convex curves of constant width'. A classical non-circular example is the Reuleaux triangle [6] which is made of three circular arcs. But a non-circular plane convex curve of constant width can be smooth, and not having any circular arc in its boundary. The notion of a convex body of constant width can of course be extended to higher dimensions. For a recent survey on the topic, we refer the reader to [2]

In this paper, we are essentially interested in non-circular algebraic curves of constant width. Rabinowitz [5] found that the zero set of the following polynomial $P \in \mathbb{R}[X, Y]$ forms a non-circular algebraic curve of constant width in $\mathbb{R}^{2}$ :

$$
\begin{aligned}
P(x, y) & :=\left(x^{2}+y^{2}\right)^{4}-45\left(x^{2}+y^{2}\right)^{3}-41283\left(x^{2}+y^{2}\right)^{2} \\
& +7950960\left(x^{2}+y^{2}\right)+16\left(x^{2}-3 y^{2}\right)^{3}+48\left(x^{2}+y^{2}\right)\left(x^{2}-3 y^{2}\right)^{2} \\
& +x\left(x^{2}-3 y^{2}\right)\left(16\left(x^{2}+y^{2}\right)^{2}-5544\left(x^{2}+y^{2}\right)+266382\right)-720^{3} .
\end{aligned}
$$


Then, he raised the following open questions: "The polynomial curve found is pretty complicated. Can it be put in simpler form? Our polynomial is of degree 8. Is there one with lower degree? What is the lowest degree polynomial whose graph is a noncircular curve of constant width?". Recently, it has been proved by Bardet and Bayen [1, Cor. 2.1] that the degree of $P$, that is 8 , is the minimum possible degree for a non-circular plane convex curve of constant width. Here, we emphasize the convexity assumption because it is implicit in the statement of Corollary 2.1 in [1]. In this short note, we provide additional answers to Rabinowitz's open questions. First, we recall the notion of a plane hedgehog curve of constant width, and we notice that in this setting, we can find algebraic curves of constant width much simpler. Second, we give an example of a non-circular smooth algebraic curve of constant width whose equation is simpler than the one of Rabinowitz. Finally, we notice that we can deduce from it (relatively) simple examples in higher dimensions.

\section{Plane algebraic hedgehogs of constant width}

Here, we will follow more or less [3].

Definition For any smooth function $h: \mathbb{S}^{1}=\mathbb{R} \backslash 2 \pi \mathbb{Z} \rightarrow \mathbb{R}, \theta \mapsto h(\theta)$, we let $\mathcal{H}_{h}$ denote the envelope of the family of lines given by

$$
x \cos \theta+y \sin \theta=h(\theta)
$$

where $(x, y)$ are the coordinates in the canonical basis of the Euclidean vector space $\mathbb{R}^{2}$. We say that $\mathcal{H}_{h}$ is the plane hedgehog with support function $h$, and that $\mathcal{H}_{h}$ is projective if $h(\theta+\pi)=-h(\theta)$ for all $\theta \in \mathbb{S}^{1}$.

Partial differentiation of (1) yields

$$
-x \sin \theta+y \cos \theta=h^{\prime}(\theta) .
$$

From (1) and (2), the parametric equations for $\mathcal{H}_{h}$ are

$$
\left\{\begin{array}{c}
x=h(\theta) \cos \theta-h^{\prime}(\theta) \sin \theta \\
y=h(\theta) \sin \theta+h^{\prime}(\theta) \cos \theta
\end{array}\right.
$$

The family of lines $(D(\theta))_{\theta \in \mathbb{S}^{1}}$ of which $\mathcal{H}_{h}$ is the envelope is the family of 'support lines' of $\mathcal{H}_{h}$. Suppose that $\mathcal{H}_{h}$ has a well defined tangent line at the point $(x, y)$, say $T$. Then $T$ is the support line with equation (1): the unit vector $u(\theta)=(\cos \theta, \sin \theta)$ is normal to $T$ and $h(\theta)$ may be interpreted as the signed distance from the origin to $T$. 
A plane hedgehog is thus simply a plane envelope that has exactly one oriented support line in each direction. A singularity-free plane hedgehog is simply a convex curve. A plane hedgehog is projective if it has exactly one nonoriented support line in each direction

Now, we can define the width, say $w_{h}(\theta)$, of such a plane hedgehog $\mathcal{H}_{h}$ in the direction $u(\theta)$ to be the signed distance between the two support lines of $\mathcal{H}_{h}$ that are orthogonal to $u(\theta)$, that is by

$$
w_{h}(\theta)=h(\theta)+h(\theta+\pi) .
$$

Thus plane projective hedgehogs are hedgehogs of constant width 0 , and the condition that a plane hedgehog $\mathcal{H}_{h}$ is of constant width $2 r$ is simply that its support function $h$ has the form $f+r$, where $f$ is the support function of a projective hedgehog. Here are three examples of plane hedgehogs: (a) a convex hedgehog of constant width; $(b)$ a hedgehog with four cusps; $(c)$ a plane projective hedgehog which is a hypocycloid with three cusps.

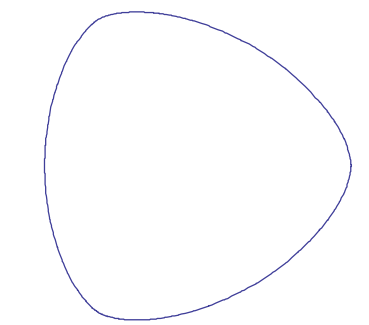

(a) $h(\theta)=10+\cos (3 \theta)$

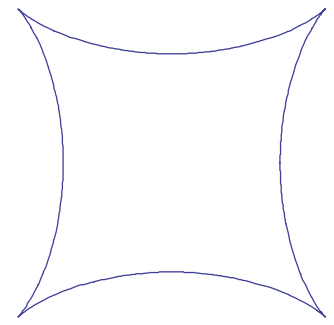

(b)

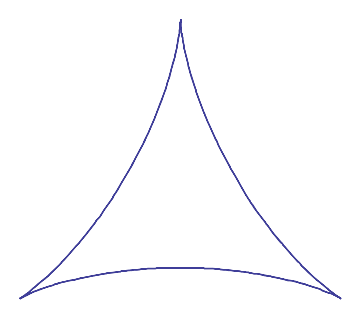

$(c)$

Figure 1

Theorem. The projective hedgehog $\mathcal{H}_{h} \subset \mathbb{R}^{2}$ with support function $h: \mathbb{S}^{1} \rightarrow \mathbb{R}, \theta \mapsto \sin (3 \theta)$ is a non-circular algebraic curve of constant width 0 with equation

$$
\left(x^{2}+y^{2}\right)^{2}+18\left(x^{2}+y^{2}\right)-8 y\left(y^{2}-3 x^{2}\right)=27 .
$$

Proof. We already know that $\mathcal{H}_{h}$ is a non-circular curve of constant width 0 . From the parametric equations for $\mathcal{H}_{h}$, we deduce that

$$
x^{2}+y^{2}=h(\theta)^{2}+h^{\prime}(\theta)^{2}=\sin ^{2}(3 \theta)+9 \cos ^{2}(3 \theta)=5+4 \cos (6 \theta) .
$$

Now, $h: \mathbb{S}^{1} \rightarrow \mathbb{R}, \theta \mapsto \sin (3 \theta)$ is the restriction of the polynomial $-y\left(y^{2}-3 x^{2}\right)$ to the unit circle $\mathbb{S}^{1}$, and the linearization of $-y\left(y^{2}-3 x^{2}\right)$ as a trigonometric function of $\theta$ gives 


$$
-y\left(y^{2}-3 x^{2}\right)=-12-14 \cos (6 \theta)-\cos (12 \theta)=-11-14 \cos (6 \theta)-2 \cos ^{2}(6 \theta) .
$$

From the above two equations, we deduce easily that

$$
\left(x^{2}+y^{2}\right)^{2}+18\left(x^{2}+y^{2}\right)-8 y\left(y^{2}-3 x^{2}\right)=27,
$$

\section{A non-circular algebraic curve of constant width whose equation is not too complicated}

Any hedgehog whose support function $h: \mathbb{S}^{1} \rightarrow \mathbb{R}$ is of the form $h(\theta)=r-\sin (3 \theta)$, for some constant $r$, is a hedgehog of constant width $2 r$. Such a function $h: \mathbb{S}^{1} \rightarrow \mathbb{R}$ is the support function of a convex body if and only if $\left(h+h^{\prime \prime}\right)(\theta)=r-8 \sin (3 \theta) \geq 0$ for all $\theta \in \mathbb{S}^{1}$, that is if and only if $r \geq 8$. We choose $r=8$ in order to be 'as closed as possible' to the previous example.

Theorem. The plane hedgehog $\mathcal{H}_{h}$ with support function $h: \mathbb{S}^{1} \rightarrow \mathbb{R}$, $\theta \mapsto 8-\sin (3 \theta)=4 \sin ^{3} \theta-3 \sin \theta+8$ is a non-circular convex algebraic curve of constant width 16 with equation

$$
\begin{aligned}
& \left(\left(x^{2}+y^{2}\right)^{2}+8 y\left(y^{2}-3 x^{2}\right)\right)^{2}+432 y\left(y^{2}-3 x^{2}\right)\left(351-10\left(x^{2}+y^{2}\right)\right) \\
& =567^{3}+28\left(x^{2}+y^{2}\right)^{3}+486\left(x^{2}+y^{2}\right)\left(67\left(x^{2}+y^{2}\right)-567 \times 18\right) .
\end{aligned}
$$

Proof. The parametric equations for $\mathcal{H}_{h}$ are equivalent to:

$$
\left\{\begin{array}{l}
x=-8\left(\sin ^{3}(\theta)-1\right) \cos (\theta) \\
y=-2 \cos (2 \theta)-\cos (4 \theta)+8 \sin (\theta) .
\end{array}\right.
$$

Expanding $x$ and $y$ in terms of $c=\cos \theta$ and $s=\sin \theta$, we obtain after simplification:

$$
\left\{\begin{array}{l}
x=-8\left(s^{3}-1\right) c \\
y=-3+4 s\left(2+3 s-2 s^{3}\right) .
\end{array}\right.
$$

Squaring the first equation and substituting in $c^{2}=1-s^{2}$ gives us the following system of equations in the three unknowns $x, y$, and $s$ :

$$
\left\{\begin{array}{l}
64\left(1-s^{2}\right)\left(s^{3}-1\right)^{2}-x^{2}=0 \\
-3+4 s\left(2+3 s-2 s^{3}\right)-y=0
\end{array}\right.
$$


We then eliminate $s$ by computing the resultant of the polynomials $A(s)=64\left(1-s^{2}\right)\left(s^{3}-1\right)^{2}-x^{2}$ and $B(s)=-3+4 s\left(2+3 s-2 s^{3}\right)-y$ with Mathematica, and find after simplification that:

$$
\begin{aligned}
& \left(\left(x^{2}+y^{2}\right)^{2}+8 y\left(y^{2}-3 x^{2}\right)\right)^{2}+432 y\left(y^{2}-3 x^{2}\right)\left(351-10\left(x^{2}+y^{2}\right)\right) \\
& =567^{3}+28\left(x^{2}+y^{2}\right)^{3}+486\left(x^{2}+y^{2}\right)\left(67\left(x^{2}+y^{2}\right)-567 \times 18\right) .
\end{aligned}
$$

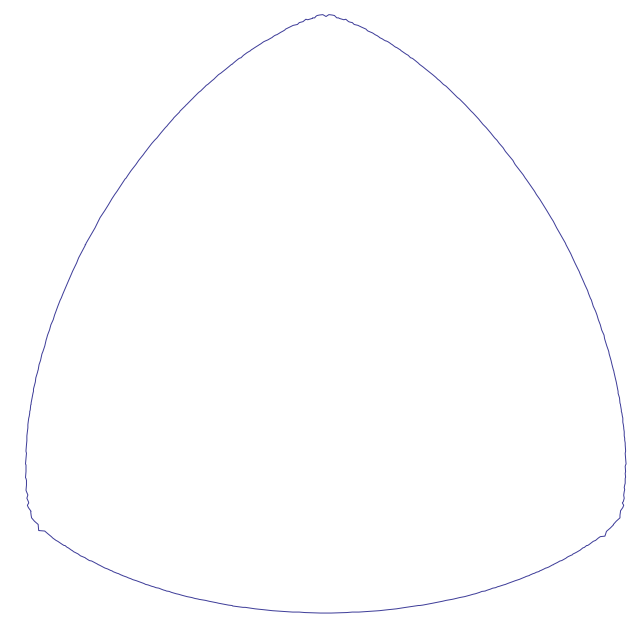

Figure 2. The non-circular convex curve of constant width 16 with equation (3)

\section{Higher dimension}

The notion of a hedgehog of constant width can of course be extended to higher dimensions (see, e.g. [4]). Each of the above two examples of algebraic curves of constant width admits an axis of symmetry in $\mathbb{R}^{2}$. By rotating it around such an axis, we deduce immediately an example of algebraic surface of revolution that is of constant width in $\mathbb{R}^{3}$. More precisely, the algebraic surface with equation

$$
\left(x^{2}+y^{2}+z^{2}\right)^{2}+18\left(x^{2}+y^{2}+z^{2}\right)-8 z\left(z^{2}-3\left(x^{2}+y^{2}\right)\right)=27
$$

is a 'projective hedgehog' of revolution and a surface of constant width 0 in $\mathbb{R}^{3}$ (see Figure 3, left), and the algebraic surface with equation 


$$
\begin{gathered}
\left(\left(x^{2}+y^{2}+z^{2}\right)^{2}+8 z\left(z^{2}-3\left(x^{2}+y^{2}\right)\right)\right)^{2} \\
+432 z\left(z^{2}-3\left(x^{2}+y^{2}\right)\right)\left(351-10\left(x^{2}+y^{2}+z^{2}\right)\right) \\
=567^{3}+28\left(x^{2}+y^{2}+z^{2}\right)^{3} \\
+486\left(x^{2}+y^{2}+z^{2}\right)\left(67\left(x^{2}+y^{2}+z^{2}\right)-567 \times 18\right)
\end{gathered}
$$

is a convex surface of constant width 16 in $\mathbb{R}^{3}$ (see Figure 3 , right).
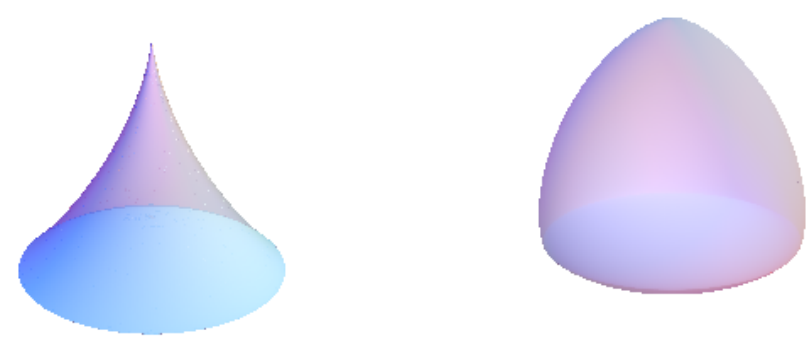

Figure 3: Our two algebraic surfaces of constant width

\section{References}

[1] M. Bardet, T. Bayen, On the degree of the polynomial defining a planar algebraic curves of constant width. arXiv 2013: https://arxiv.org/abs/1312.4358

[2] H. Martini, L. Montejano, D. Oliveros, Bodies of constant width. An introduction to convex geometry with applications. Birkhäuser (2019)

[3] Y. Martinez-Maure, A Note on the Tennis Ball Theorem. Amer. Math. Monthly 103 (1996), 338-340.

[4] Y. Martinez-Maure, A stability estimate for the AleksandrovFenchel inequality under regularity assumptions. Monatsh. Math. 182 (2017), 65-76.

[5] S. Rabinowitz, A polynomial curve of constant width. Missouri J. Math. Sci 9 (1997), 23-27.

[6] Reuleaux triangle: en.wikipedia.org/wiki/Reuleaux_triangle

Y. Martinez-Maure

Sorbonne Université et Université de Paris

Institut Mathématique de Jussieu - Paris Rive Gauche

UMR 7586 du CNRS

Bâtiment Sophie Germain

Case 7012 
75205 Paris Cedex 13

France 